\title{
Complexity of Road Coloring with Prescribed Reset Words
}

\author{
Vojtěch Vorel ${ }^{1 \star}$ and Adam Roman ${ }^{2 \star \star}$ \\ 1 Faculty of Mathematics and Physics, Charles University, Malostranské nám. 25, \\ Prague, Czech Republic, \\ vorel@ktiml.mff.cuni.cz, \\ 2 Institute of Computer Science, Jagiellonian University, Lojasiewicza 6, 30-348 \\ Krakow, Poland, \\ roman@ii.uj.edu.pl
}

\begin{abstract}
By the Road Coloring Theorem (Trahtman, 2008), the edges of any given aperiodic directed multigraph with a constant out-degree can be colored such that the resulting automaton admits a reset word. There may also be a need for a particular reset word to be admitted. For certain words it is NP-complete to decide whether there is a suitable coloring. For the binary alphabet, we present a classification that separates such words from those that make the problem solvable in polynomial time. The classification differs if we consider only strongly connected multigraphs. In this restricted setting the classification remains incomplete.
\end{abstract}

Keywords: Algorithms on Automata and Words, Road Coloring Theorem, Road Coloring Problem, Reset Word, Synchronizing Word

\section{Introduction}

Questions about synchronization of finite automata have been studied since the early times of automata theory. The basic concept is very natural: we want to find an input sequence that would get a given machine to a unique state, no matter in which state the machine was before. Such sequence is called a reset word. If an automaton has a reset word, we call it a synchronizing automaton.

In the study of road coloring, synchronizing automata are created from directed multigraphs through edge coloring. A directed multigraph is said to be admissible, if it is aperiodic and has a constant out-degree. A multigraph needs to be admissible in order to have a synchronizing coloring. Given an alphabet $I$ and an admissible graph with out-degrees $|I|$, the following questions arise:

1. Is there a coloring such that the resulting automaton has a reset word?

2. Given a number $k \geq 1$, is there a coloring such that the resulting automaton has a reset word of length at most $k$ ?

\footnotetext{
* Supported by the Czech Science Foundation grant GA14-10799S.

** Supported in part by Polish MNiSW grant IP 2012052272.
} 
3. Given a word $w \in I^{\star}$, is there a coloring such that $w$ is a reset word of the resulting automaton?

4. Given a set of words $W \subseteq I^{\star}$, is there a coloring such that some $w \in W$ is a reset word of the resulting automaton?

For the first question it was conjectured in 1977 by Adler, Goodwyn, and Weiss [1] that the answer is always yes. The conjecture was known as the Road Coloring Problem until Trahtman [5] in 2008 found a proof, turning the claim into the Road Coloring Theorem.

The second question was initially studied in the paper [3] presented at LATA 2012, while the yet-unpublished papers [2] and 6] give closing results: The problem is NP-complete for any fixed $k \geq 4$ and any fixed $|I| \geq 2$. The instances with $k \leq 3$ or $|I|=1$ can be solved by a polynomial-time algorithm.

The third question is the subject of the present paper. We show that the problem becomes NP-complete even if restricted to $|I|=2$ and $w=a b b$ or to $|I|=2$ and $w=a b a$, which may seem surprising. Moreover, we provide a complete classification of binary words: The NP-completeness holds for $|I|=2$ and any $w \in\{a, b\}^{\star}$ that does not equal $a^{k}, b^{k}, a^{k} b$, nor $b^{k} a$ for any $k \geq 1$. On the other hand, for any $w$ that matches some of these patterns, the restricted problem is solvable in polynomial time.

The fourth question was raised in [2] and it was emphasized that there are no results about the problem. Our results about the third problem provide an initial step for this direction of research.

It is an easy but important remark that the Road Coloring Theorem holds generally if and only if it holds for strongly connected graphs. It may seem that strong connectivity can be safely assumed even if dealing with other problems related to road coloring. Surprisingly, we show that this does not hold for complexity issues. If $\mathrm{P}$ is not equal to NP, the complexity of the third problem for strongly connected graphs differs from the basic third problem in the case of $w=a b b$. However, for the strongly connected case we are not able to provide a complete characterization as described above, we give only partial results.

Due to the page limit, some proofs are omitted or shortened. The results are presented in Sections 3 and 4 .

\section{Preliminaries}

\subsection{Automata and Synchronization}

For $u, w \in I^{\star}$ we say that $u$ is a prefix, a suffix, or a factor of $w$ if $w=u v$, $w=v u$, or $w=v u v^{\prime}$ for some $v, v^{\prime} \in I^{\star}$, respectively.

A deterministic finite automaton is a triple $A=(Q, I, \delta)$, where $Q$ and $I$ are finite sets and $\delta$ is an arbitrary mapping $Q \times I \rightarrow Q$. Elements of $Q$ are called states, $I$ is the alphabet. The transition function $\delta$ can be naturally extended to $Q \times I^{\star} \rightarrow Q$, still denoted by $\delta$, slightly abusing the notation. We extend it also by defining

$$
\delta(S, w)=\{\delta(s, w) \mid s \in S\}
$$


for each $S \subseteq Q$ and $w \in I^{\star}$. If $A=(Q, I, \delta)$ is fixed, we write $r \stackrel{x}{\longrightarrow} s$ instead of $\delta(r, x)=s$.

For a given automaton $A=(Q, I, \delta)$, we call $w \in I^{\star}$ a reset word if $|\delta(Q, w)|=$ 1 . If such a word exists, we call the automaton synchronizing. Note that each word having a reset word as a factor is also a reset word.

\section{$2.2 \quad$ Road Coloring}

In the rest of the paper we use the term graph for a directed multigraph. A graph is:

1. aperiodic, if 1 is the only common divisor of all the lengths of cycles,

2. admissible, if it is aperiodic and all its out-degrees are equal,

3. road colorable, if its edges can be labeled such that a synchronized deterministic finite automaton arises.

Naturally, we identify a coloring of edges with a transition function $\delta$ of the resulting automaton. It is not hard to observe that any road colorable graph is admissible. In 1977 Adler, Goodwyn, and Weiss [1] conjectured that the backward implication holds as well. Their question became known as the Road Coloring Problem and a positive answer was given in 2008 by Trahtman [5].

For any alphabet $I$ and $w \in I^{\star}$, by $\mathbb{G}_{w}^{|I|}$ we denote the set of graphs with all out-degrees equal to $|I|$ such that there exists a coloring $\delta$ with $|\delta(Q, w)|=1$. In this paper we work with the following computational problem:

SRCW (Synchronizing road coloring with prescribed reset words)

Input: $\quad$ Alphabet $I$, graph $G=(Q, E)$ with out-degrees $|I|, W \subseteq I^{\star}$

Output: $\quad$ Is there a $w \in W$ such that $G \in \mathbb{G}_{w}^{|I|}$ ?

In this paper we study the restrictions to one-element sets $W$, which means that we consider the complexity of the sets $\mathbb{G}_{w}^{|I|}$ themselves.

Restrictions are denoted by subscripts and superscripts: $\mathrm{SRCW}_{k, X}^{\mathcal{M}}$ denotes SRCW restricted to inputs with $|I|=k, W=X$, and $G \in \mathcal{M}$, where $\mathcal{M}$ is a class of graphs. By $\mathcal{S C}$ we denote the class of strongly connected graphs. Having a graph $G=(Q, E)$ fixed, by $\mathrm{d}_{G}(s, t)$ we denote the length of shortest directed path from $s \in Q$ to $t \in Q$ in $G$. For each $k \geq 0$ we denote

$$
V_{k}(q)=\left\{s \in Q \mid \mathrm{d}_{G}(s, q)=k\right\} .
$$

Having $R \subseteq Q$, let $G[R]$ denote the induced subgraph of $G$ on the vertex set $R$. If a graph $G$ has constant out-degree $|I|$, a vertex $v \in Q$ is called a sink state if there are $|I|$ loops on $v$. By $\mathcal{Z}$ we denote the class of graphs having a sink state. The following lemma can be easily proved by a reduction that adds a chain of $|u|$ new states to each state of a graph:

Lemma 1. Let $|I| \geq 1$ and $u, w \in\{a, b\}^{\star}$. Then:

1. If $\mathrm{SRCW}_{k,\{w\}}$ is NP-complete, so is $\mathrm{SRCW}_{k,\{u w\}}$.

2. If $\mathrm{SRCW}_{k,\{w\}}^{\mathcal{Z}}$ is NP-complete, so is $\mathrm{SRCW}_{k,\{u w\}}^{\mathcal{Z}}$. 


\section{A Complete Classification of Binary Words According to Complexity of $\mathrm{SRCW}_{2,\{w\}}$}

The theorem below presents one of the main results of the present paper. Assuming that $\mathrm{P}$ does not equal NP, it introduces an exact dichotomy concerning the words over binary alphabets. Let us fix the following partition of $\{a, b\}^{\star}$ :

$$
\begin{array}{ll}
T_{1}=\left\{a^{k}, b^{k} \mid k \geq 0\right\}, & T_{3}=\left\{a^{l} b^{k}, b^{l} a^{k} \mid k \geq 2, l \geq 1\right\}, \\
T_{2}=\left\{a^{k} b, b^{k} a \mid k \geq 1\right\}, & T_{4}=\{a, b\}^{\star} \backslash\left(T_{1} \cup T_{2} \cup T_{3}\right) .
\end{array}
$$

For the NP-completeness reductions throughout the present paper we use a suitable variant of the satisfiability problem. The following can be verified using the Schaefer's dichotomy theorem [4]:

Lemma 2. It holds that W-SAT is NP-complete.

W-SAT

Input: $\quad$ Finite set $X$ of variables, finite set $\Phi \subseteq X^{4}$ of clauses.

Output: $\quad$ Is there an assignment $\xi: X \rightarrow\{\mathbf{0}, \mathbf{1}\}$ such that for each clause $\left(z_{1}, z_{2}, z_{3}, z_{4}\right) \in \Phi$ it holds that:

(1) $\xi\left(z_{i}\right)=\mathbf{1}$ for some $i$,

(2) $\xi\left(z_{i}\right)=\mathbf{0}$ for some $i \in\{1,2\}$,

(3) $\xi\left(z_{i}\right)=\mathbf{0}$ for some $i \in\{3,4\}$ ?

In this section we use reductions from W-SAT to prove the NP-completeness of $\mathrm{SRCW}_{2,\{w\}}$ for each $w \in T_{3}$ and $w \in T_{4}$. In the case of $w \in T_{4}$ the reduction produces only graphs having sink states. This shows that for $w \in T_{4}$ the problem $\mathrm{SRCW}_{2,\{w\}}^{\mathcal{Z}}$ is NP-complete as well, which turns out to be very useful in Section 4. where we deal with strongly connected graphs. For $w \in T_{3}$ we also prove NP-completeness, but we use automata without sink states. We show that the cases with $w \in T_{1} \cup T_{2}$ are decidable in polynomial time.

In all the figures below we use bold solid arrows and bold dotted arrows for the letters $a$ and $b$ respectively.

Theorem 3. Let $w \in\{a, b\}^{\star}$.

1. If $w \in T_{1} \cup T_{2}$, the problem $\mathrm{SRCW}_{2,\{w\}}$ is solvable in polynomial time.

2. If $w \in T_{3} \cup T_{4}$, the problem $\mathrm{SRCW}_{2,\{w\}}$ is $N P$-complete. Moreover, if $w \in T_{4}$, the problem $\mathrm{SRCW}_{2,\{w\}}^{\mathcal{Z}}$ is NP-complete.

Proof for $w \in T_{1}$. It is easy to see that $G \in \mathbb{G}_{a^{k}}$ if and only if there is $q_{0} \in Q$ such that there is a loop on $q_{0}$ and for each $s \in Q$ we have $\mathrm{d}_{G}\left(s, q_{0}\right) \leq k$. 
Proof for $w \in T_{2}$. For a fixed $q_{0} \in Q$, we denote $Q_{1}=\left\{s \in Q \mid s \longrightarrow q_{0}\right\}$ and

$$
R=\left\{s \in Q_{1} \mid H_{1} \text { has a cycle reachable from } s\right\},
$$

where $H_{1}$ is obtained from $G\left[Q_{1}\right]$ by decreasing multiplicity by 1 for each edge ending in $q_{0}$. If $q_{0} \notin Q_{1}$, we have $H_{1}=G\left[Q_{1}\right]$. Let us prove that $G \in \mathbb{G}_{a^{k} b}$ if and only if there is $q_{0} \in Q$ such that:

1. It holds that $\mathrm{d}_{G}\left(s, q_{0}\right) \leq k+1$ for each $s \in Q$.

2. For each $s \in Q$ there is a $q \in R$ such that $\mathrm{d}_{G}(s, q) \leq k$.

First, check the backward implication. For each $r \in R$, we color by $b$ an edge of the form $r \longrightarrow q_{0}$ that does not appear in $H_{1}$. Then we fix a forest of shortest paths from all the vertices of $Q \backslash R$ into $R$. Due to the second condition above, the branches have length at most $k$. We color by $a$ the edges used in the forest. We have completely specified a coloring of edges. Now, for any $s \in Q$ a prefix $a^{j}$ of $a^{k} b$ takes us into $R$, the factor $a^{k-j}$ keeps us inside $R$, and with the letter $b$ we end up in $q_{0}$.

As for the forward implication, the first condition is trivial. For the second one, take any $s \in Q$ and denote $s_{j}=\delta\left(s, a^{j}\right)$ for $j \geq 0$. Clearly, $s_{k} \in Q_{1}$, but we show also that $s_{k} \in R$, so we can set $q=s_{k}$ in the last condition. Indeed, whenever $s_{j} \in Q_{1}$ for $j \geq k$, we remark that $\delta\left(s_{j-k+1}, a^{k}\right)=q_{0}$ and thus $s_{j+1} \in Q_{1}$ as well. Since $j$ can grow infinitely, there is a cycle within $Q_{1}$ reachable from $s_{k}$.

Proof for $w \in T_{3}$. Due to Lemma 1, it is enough to deal with $w=a b^{k}$ for each $k \geq 2$. For a polynomial-time reduction from W-SAT, take an instance $X=\left\{x_{1}, \ldots, x_{n}\right\}, \Phi=\left\{C_{1}, \ldots, C_{m}\right\}$, where $C_{j}=\left(z_{j, 1}, z_{j, 2}, z_{j, 3}, z_{j, 4}\right)$ for each $j=1, \ldots, m$. We build the graph $G_{k, \phi}=(Q, E)$ defined by Fig. 1 Note that:

- In Fig. 11 states are represented by discs. For each $j=1, \ldots, m$, the edges outgoing from $\mathrm{C}_{i}^{\prime}$ and $\mathrm{C}_{i}^{\prime \prime}$ represent the formula $\Phi$ by leading to the states $z_{j, 1}, z_{j, 2}, z_{j, 3}, z_{j, 4} \in\left\{x_{1}, \ldots, x_{n}\right\} \subseteq Q$.

- In the case of $k=2$ the state $\mathrm{V}_{i, 2}$ does not exist, so we set $x_{i} \longrightarrow \mathrm{D}_{0}$ and $\mathrm{V}_{i, 1} \longrightarrow \mathrm{D}_{0}$ instead of $x_{i} \longrightarrow \mathrm{V}_{i, 2}$ and $\mathrm{V}_{i, 1} \longrightarrow \mathrm{V}_{i, 2}$.

We show that $G_{k, \Phi} \in \mathbb{G}_{a b^{k}}$ if and only if there is an assignment $\xi: X \rightarrow\{\mathbf{0}, \mathbf{1}\}$ satisfying the conditions given by $\Phi$.

First, let there be a coloring $\delta$ of $G_{k, \Phi}$ such that $\left|\delta\left(Q, a b^{k}\right)\right|=1$. Observe that necessarily $\delta\left(Q, a b^{k}\right)=\left\{\mathrm{D}_{0}\right\}$, while there is no loop on $\mathrm{D}_{0}$. We use this fact to observe that whenever $x_{i} \in \delta(Q, a)$, the edges outgoing from $x_{i}, \mathrm{~V}_{i, 1}, \ldots, \mathrm{V}_{i, k-1}$ must be colored according to Fig. 2, but if $x_{i} \in \delta(Q, a b)$, then they must be colored according to Fig. 3, Let $\xi\left(x_{i}\right)=\mathbf{1}$ if $x_{i} \in \delta(Q, a b)$ and $\xi\left(x_{i}\right)=\mathbf{0}$ otherwise. Choose any $j \in\{1, \ldots, m\}$ and observe that

$$
\xi\left(\delta\left(\mathrm{C}_{j}, a b\right)\right)=\mathbf{1}, \quad \xi\left(\delta\left(\mathrm{C}_{j}^{\prime}, a\right)\right)=\mathbf{0}, \quad \xi\left(\delta\left(\mathrm{C}_{j}^{\prime \prime}, a\right)\right)=\mathbf{0},
$$

thus we can conclude that all the conditions from the definition of W-SAT hold for the clause $C_{j}$. 

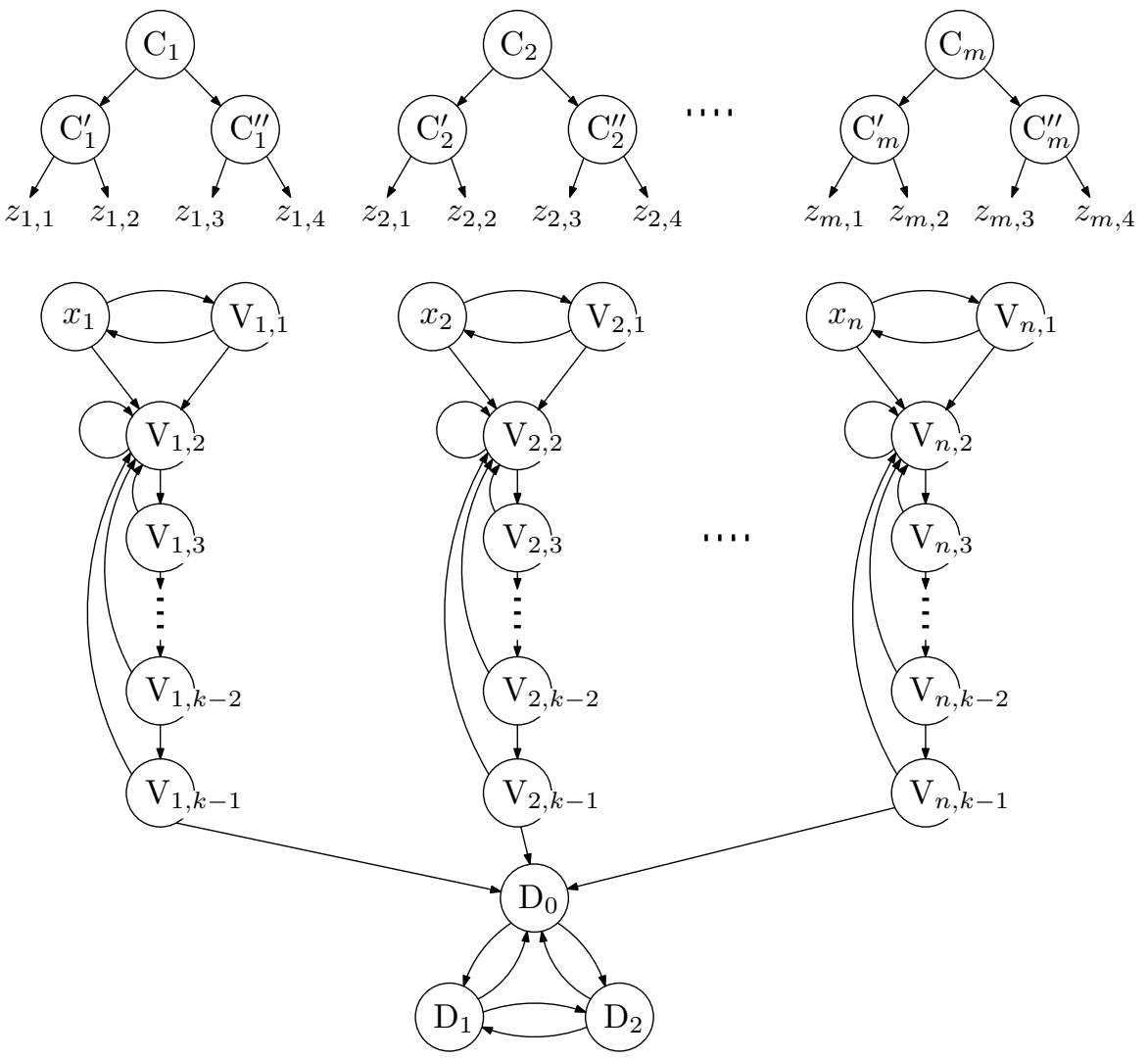

Fig. 1. The graph $G_{k, \Phi}$ reducing W-SAT to $\mathrm{SRCW}_{|I|=2, W=\left\{a b^{k}\right\}}$ for $k \geq 2$

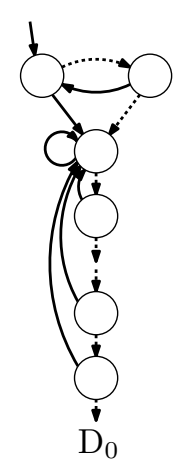

Fig. 2. A coloring corresponding to $\xi\left(x_{i}\right)=\mathbf{0}$

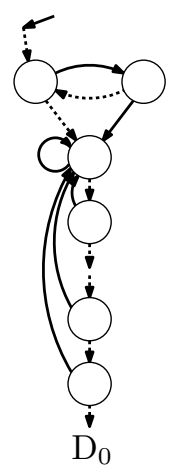

Fig. 3. A coloring corresponding to $\xi\left(x_{i}\right)=\mathbf{1}$
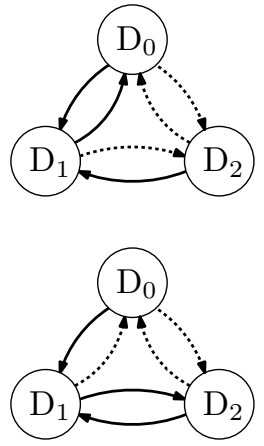

Fig. 4. Colorings for $k$ even (top) and odd (bottom) 
On the other hand, let $\xi$ be a satisfying assignment of $\Phi$. For each $j$ we color the edges outgoing from $\mathrm{C}_{j}, \mathrm{C}_{j}^{\prime}, \mathrm{C}_{j}^{\prime \prime}$ such that the $a b$-path from $\mathrm{C}_{j}$ leads to the $z_{j, i}$ with $\xi\left(z_{j, i}\right)=1$ and the $a$-paths from $\mathrm{C}_{j}^{\prime}, \mathrm{C}_{j}^{\prime \prime}$ lead to the $z_{j, i^{\prime}}$ and $z_{j, i^{\prime \prime}}$ with $\xi\left(z_{j, i^{\prime}}\right)=\mathbf{0}, \xi\left(z_{j, i^{\prime \prime}}\right)=\mathbf{0}$, where $i^{\prime} \in\{1,2\}, i^{\prime \prime} \in\{3,4\}$. For the edges outgoing from $x_{i}, \mathrm{~V}_{i, 1}, \ldots, \mathrm{V}_{i, k-1}$ we use Fig. 2 if $\xi\left(x_{i}\right)=\mathbf{0}$ and Fig. 3 if $\xi\left(x_{i}\right)=\mathbf{1}$. The transitions within $\mathrm{D}_{0}, \mathrm{D}_{1}, \mathrm{D}_{2}$ are colored according to Fig. 4, depending on the parity of $k$. Observe that for each $i \in\{1, \ldots, n\}$ we have $x_{i} \notin \delta(Q, a b)$ if $\xi\left(x_{i}\right)=\mathbf{0}$ and $x_{i} \notin \delta(Q, a)$ if $\xi\left(x_{i}\right)=\mathbf{1}$. Using this fact we check that $\delta(Q, w)=\left\{\mathrm{D}_{0}\right\}$.

Proof for $w \in T_{4}$. Any $w \in T_{4}$ can be written as $w=v a^{j} b^{k} a^{l}$ or $w=v b^{j} a^{k} b^{l}$ for $j, k, l \geq 1$. Due to Lemma 1 it is enough to deal with $w=a b^{k} a^{l}$ for each $k, l \geq 1$. Take an instance of W-SAT as above and construct the graph $G_{w, \Phi}=(Q, E)$ defined by Fig. 5 . Note that:

- In the case of $l=1$, the state $\mathrm{Z}_{i, 1}$ does not exist, so we set $\mathrm{W}_{i}^{\prime} \longrightarrow \mathrm{D}_{0}$ and $\mathrm{V}_{i, k-1} \longrightarrow \mathrm{D}_{0}$ instead of $\mathrm{W}_{i}^{\prime} \longrightarrow \mathrm{Z}_{i, 1}$ and $\mathrm{V}_{i, k-1} \longrightarrow \mathrm{Z}_{i, 1}$.

- In the case of $k=1$, the state $V_{i, 1}$ does not exist, so we set $x_{i} \longrightarrow \mathrm{Z}_{i, 1}$ (or $x_{i} \longrightarrow \mathrm{D}_{0}$ if $l=1$ ) and $x_{i} \longrightarrow \mathrm{W}_{i}$ instead of $x_{i} \rightrightarrows \mathrm{V}_{i, 1}$.
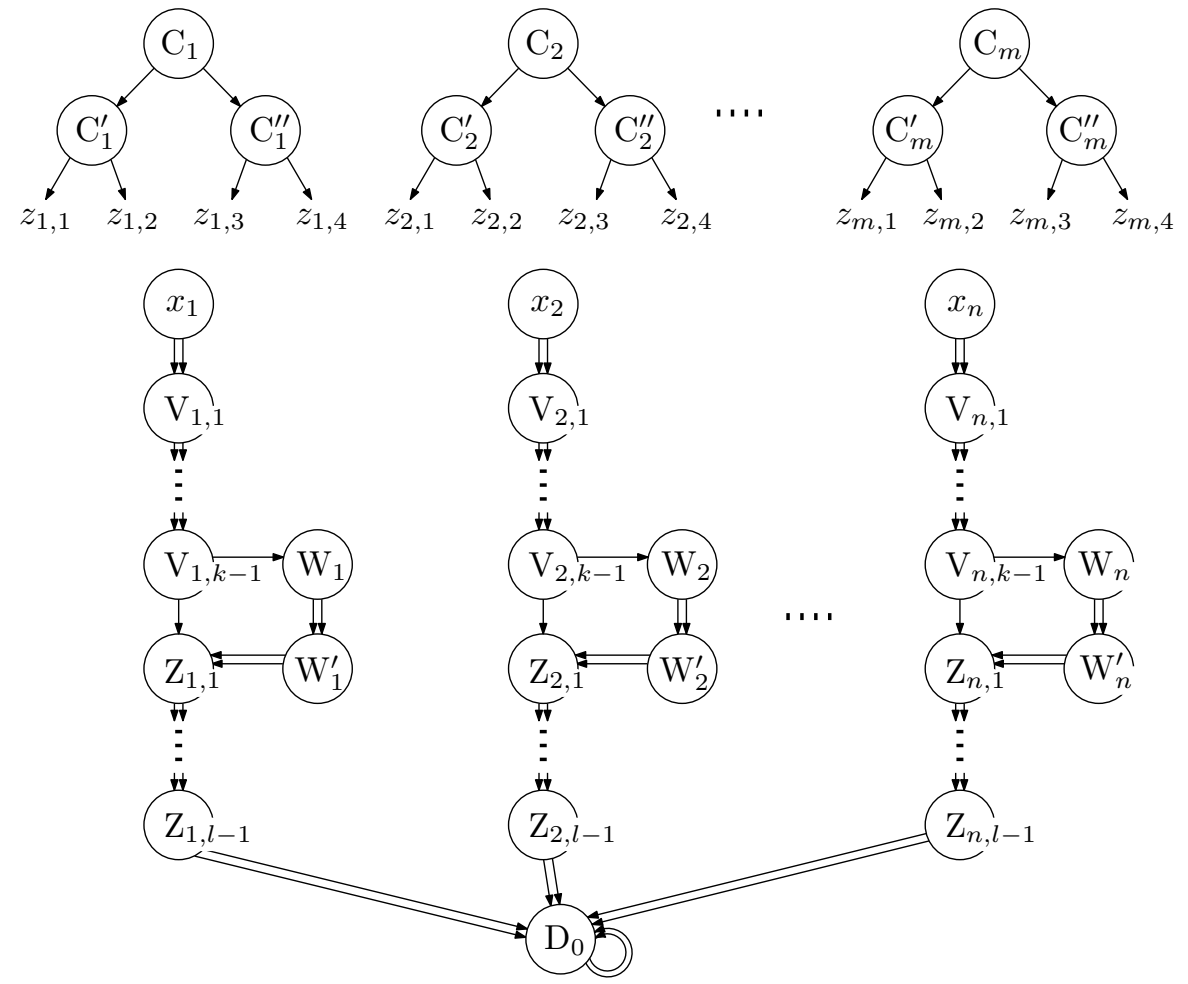

Fig. 5. The graph $G_{w, \Phi}$ reducing W-SAT to $\operatorname{SRCW}_{|I|=2, W=\left\{a b^{k} a^{l}\right\}}^{\mathcal{Z}}$ for $k, l \geq 1$ 
Let there be a coloring $\delta$ of $G_{w, \Phi}$ such that $|\delta(Q, w)|=1$. Observe that $\delta(Q, w)=$ $\left\{\mathrm{D}_{0}\right\}$. Next, observe that whenever $x_{i} \in \delta(Q, a)$, then $\mathrm{V}_{i, k-1} \stackrel{b}{\longrightarrow} \mathrm{Z}_{i, 1}$, but if $x_{i} \in \delta(Q, a b)$, then $\mathrm{V}_{i, k-1} \stackrel{a}{\longrightarrow} \mathrm{Z}_{i, 1}$. Let $\xi\left(x_{i}\right)=\mathbf{1}$ if $x_{i} \in \delta(Q, a b)$ and $\xi\left(x_{i}\right)=\mathbf{0}$ otherwise. We choose any $j \in\{1, \ldots, m\}$ and conclude exactly as we did in the case of $T_{3}$.

On the other hand, let $\xi$ be a satisfying assignment of $\Phi$. For each $j$, we color the edges outgoing from $\mathrm{C}_{j}, \mathrm{C}_{j}^{\prime}, \mathrm{C}_{j}^{\prime \prime}$ as we did in the case of $T_{3}$. For each $i$, we put $\mathrm{V}_{i, k-1} \stackrel{a}{\longrightarrow} \mathrm{Z}_{i, 1}, \mathrm{~V}_{i, k-1} \stackrel{b}{\longrightarrow} \mathrm{W}_{i}$ if $\xi\left(x_{i}\right)=\mathbf{1}$ and the reversed variant if $\xi\left(x_{i}\right)=\mathbf{0}$.

\section{A Partial Classification of Binary Words According to Complexity of $\mathrm{SRCW}_{2,\{w\}}^{\mathcal{S C}}$}

Clearly, for any $w \in T_{1} \cup T_{2}$ we have $\operatorname{SRCW}_{2,\{w\}}^{\mathcal{S C}} \in \mathrm{P}$. In Section 4.1 we show that

$$
\mathrm{SRCW}_{2,\{a b b\}}^{\mathcal{S C}} \in \mathrm{P}
$$

which is a surprising result because the general $\mathrm{SRCW}_{2,\{w\}}$ is NP-complete for any $w \in T_{3}$, including $w=a b b$. We are not aware of any other words that witness this difference between SRCW ${ }^{\mathcal{S C}}$ and SRCW.

In Section 4.2 we introduce a general method using sink devices that allows us to prove the NP-completeness of $\mathrm{SRCW}_{2,\{w\}}^{\mathcal{S C}}$ for infinitely many words $w \in T_{4}$, including any $w \in T_{4}$ with the first and last letter being the same. However, we are not able to apply the method to each $w \in T_{4}$.

\subsection{A Polynomial-Time Case}

A graph $G=(Q, E)$ is said to be $k$-lifting if there exists $q_{0} \in Q$ such that for each $s \in Q$ there is an edge leading from $s$ into $V_{k}\left(q_{0}\right)$. Instead of 2-lifting we just say lifting.

Lemma 4. If $G$ is a k-lifting graph, then $G \in \mathbb{G}_{a b^{k}}$.

Lemma 5. If $G$ is strongly connected, $G$ is not lifting, and $G \in \mathbb{G}_{a b b}$ via $\delta$ and $q_{0}$, then $\delta$ has no b-transition ending in $V_{2}\left(q_{0}\right) \cup V_{3}\left(q_{0}\right)$. Moreover, $V_{3}\left(q_{0}\right)=\emptyset$.

Proof. First, suppose for a contradiction that some $s \in V_{2}\left(q_{0}\right) \cup V_{3}\left(q_{0}\right)$ has an incoming $b$-transition. Together with its outgoing $b$-transition we have

$$
r \stackrel{b}{\longrightarrow} s \stackrel{b}{\longrightarrow} t,
$$

where $s \neq q_{0}$ and $t \neq q_{0}$. Due to the strong connectivity there is a shortest path $P$ from $q_{0}$ to $r$ (possibly of length 0 if $r=q_{0}$ ). The path $P$ is made of $b$-transitions. Indeed, if there were some $a$-transitions, let $r^{\prime} \stackrel{a}{\longrightarrow} r^{\prime \prime}$ be the last one. The $a b b$-path outgoing from $r^{\prime}$ ends in $\delta\left(r^{\prime \prime}, b b\right)$, which either lies on $P$ or in $\{s, t\}$, so it is different from $q_{0}$ and we get a contradiction. 
It follows that $\delta\left(q_{0}, b\right) \neq q_{0}$ and $\delta\left(q_{0}, b b\right) \neq q_{0}$, so there cannot be any $a$ transition incoming to $q_{0}$. Hence for any $s \in V_{1}\left(q_{0}\right)$ there is a transition $s \stackrel{b}{\longrightarrow} q_{0}$ and thus there is no $a$-transition ending in $V_{1}\left(q_{0}\right)$. Because there is also no $a$ transition ending in $V_{3}\left(q_{0}\right)$, all the $a$-transitions end in $V_{2}\left(q_{0}\right)$ and thus $G$ is lifting, which is a contradiction.

Second, we show that $V_{3}\left(q_{0}\right)$ is empty. Suppose that $s \in V_{3}\left(q_{0}\right)$. No $a$ transition comes to $s$ since there is no path of length 2 from $s$ to $q_{0}$. Thus, $s$ has no incoming transition, which contradicts the strong connectivity.

Theorem 6. $\mathrm{SRCW}_{2,\{a b b\}}^{\mathcal{S C}}$ is decidable in polynomial time.

Proof. As the input we have a strongly connected $G=(Q, E)$. Suppose that $q_{0}$ is fixed (we can just try each $q_{0} \in Q$ ) and so we should decide if there is some $\delta$ with $\delta(Q, a b b)=\left\{q_{0}\right\}$. First we do some preprocessing:

- If $G$ is lifting, according to Lemma 4 we accept.

- If $V_{3}\left(q_{0}\right) \neq \emptyset$, according to Lemma 5 we reject.

- If there is a loop on $q_{0}$, we accept, since due to $V_{3}\left(q_{0}\right)=\emptyset$ we have $G \in \mathbb{G}_{b b}$.

If we are still not done, we try to find some labeling $\delta$, assuming that none of the three conditions above holds. We deduce two necessary properties of $\delta$. First, Lemma 5 says that we can safely label all the transitions ending in $V_{2}\left(q_{0}\right)$ by $a$. Second, we have $q_{0} \in \delta(Q, a)$. Indeed, otherwise all the transitions incoming to $q_{0}$ are labeled by $b$, and there cannot be any $a$-transition ending in $V_{1}\left(q_{0}\right)$ because we know that the $b$-transition outgoing from $q_{0}$ is not a loop. Thus $G$ is lifting, which is a contradiction.

Let the sets $B_{1}, \ldots, B_{\beta}$ denote the connected components (not necessarily strongly connected) of $G\left[V_{1}\left(q_{0}\right)\right]$. Note that maximum out-degree in $G\left[V_{1}\left(q_{0}\right)\right]$ is 1. Let $e=(r, s), e^{\prime}=(s, t)$ be consecutive edges with $s, t \in V_{1}\left(q_{0}\right)$ and $r \in Q$. Then the labeling $\delta$ has to satisfy

$$
e \text { is labeled by } a \Leftrightarrow e^{\prime} \text { is labeled by } b \text {. }
$$

Indeed:

- The left-to-right implication follows easily from the fact that there is no loop on $q_{0}$.

- As for the other one, suppose for a contradiction that both $e^{\prime}, e$ are labeled by $b$. We can always find a path $P$ (possibly trivial) that starts outside $V_{1}\left(q_{0}\right)$ and ends in $r$. Let $\bar{r}$ be the last vertex on $P$ that lies in $\delta(Q, a)$. Such vertex exists because we have $V_{2}\left(q_{0}\right) \cup\left\{q_{0}\right\} \subseteq \delta(Q, a)$ and $V_{3}\left(q_{0}\right)=\emptyset$. Now we can deduce that $\delta(\bar{r}, b b) \neq q_{0}$, which is a contradiction.

It follows that for each $B_{i}$ there are at most two possible colorings of its inner edges (fix variant $\mathbf{0}$ and variant $\mathbf{1}$ arbitrarily). Moreover, a labeling of any edge incoming to $B_{i}$ enforces a particular variant for whole $B_{i}$.

Let the set $A$ contain the vertices $s \in V_{2}\left(q_{0}\right) \cup\left\{q_{0}\right\}$ whose outgoing transitions lead both into $V_{1}\left(q_{0}\right)$. Edges that start in vertices of $\left(V_{2}\left(q_{0}\right) \cup\left\{q_{0}\right\}\right) \backslash A$ have only 
one possible way of coloring due to Lemma 5 , while for each vertex of $A$ there are two possibilities. Now any possible coloring can be described by $|A|+\beta$ Boolean propositions:

$$
\begin{aligned}
\mathbf{x}_{s} & \equiv e_{s} \text { is labeled by } a \\
\mathbf{y}_{B} & \equiv B \text { is labeled according to variant } \mathbf{1}
\end{aligned}
$$

for each $s \in A$ and $B \in\left\{B_{1}, \ldots, B_{\beta}\right\}$, where $e_{s}$ is a particular edge outgoing from $s$. Moreover, the claim $\delta(Q, a b b)=\left\{q_{0}\right\}$ can be equivalently formulated as a conjunction of implications of the form $\mathbf{x}_{s} \rightarrow \mathbf{y}_{B}$, so we reduce the problem to 2-SAT.

\section{$4.2 \quad$ NP-Complete Cases}

We introduce a method based on sink devices to prove the NP-completeness for a wide class of words even under the restriction to strongly connected graphs.

In the proofs below we use the notion of a partial finite automaton (PFA), which can be defined as a triple $P=(Q, I, \delta)$, where $Q$ is a finite set of states, $I$ is a finite alphabet, and $\delta$ is a partial function $Q \times I \rightarrow Q$ which can be naturally extended to $Q \times I^{\star} \rightarrow Q$. Again, we write $r \stackrel{x}{\longrightarrow} s$ instead of $\delta(r, x)=s$. We say that a PFA is incomplete if there is some undefined value of $\delta$. A sink state in a PFA has a defined loop for each letter.

Definition 7. Let $w \in\{a, b\}^{\star}$. We say that a PFA $B=(Q,\{a, b\}, \delta)$ is a sink device for $w$, if there exists $q_{0} \in Q$ such that:

1. $\delta\left(q_{0}, u\right)=q_{0}$ for each prefix $u$ of $w$,

2. $\delta(s, w)=q_{0}$ for each $s \in Q$.

Note that the trivial automaton consisting of a single sink state is a sink device for any $w \in\{a, b\}^{\star}$. However, we are interested in strongly connected sink devices that are incomplete. In Lemma 8 we show how to prove the NP-completeness using a non-specific sink device in the general case of $w \in T_{4}$ and after that we construct explicit sink devices for a wide class of words from $T_{4}$.

Lemma 8. Let $w \in T_{4}$ and assume that there exists a strongly connected incomplete sink device $B$ for $w$. Then $\mathrm{SRCW}_{2,\{w\}}^{\mathcal{S C}}$ is NP-complete.

Proof. We assume that $w$ starts by $a$ and write $w=a^{\alpha} b^{\beta} a u$ for $\alpha, \beta \geq 1$ and $u \in\{a, b\}^{\star}$. Denote $B=\left(Q_{B},\{a, b\}, \delta_{B}\right)$. For a reduction from W-SAT, take an instance $X, \Phi$ with the notation used before, assuming that each $x \in X$ occurs in $\Phi$. We construct a graph $\bar{G}_{w, \Phi}=(\bar{Q}, \bar{E})$ as follows. Let $q_{1} \in Q_{B}$ have an undefined outgoing transition, and let $B^{\prime}$ be an automaton obtained from $B$ by arbitrarily defining all the undefined transitions except for one transition outgoing from $q_{1}$. Let $G_{B^{\prime}}$ be the underlying graph of $B^{\prime}$. By Theorem 3, $\operatorname{SRCW}_{2,\{w\}}^{\mathcal{Z}}$ is NP-complete, so it admits a reduction from W-SAT. Let $G_{w, \Phi}=(Q, E)$ be the graph obtained from such reduction, removing the loop on the sink state $q_{0}^{\prime} \in Q$. Let $s_{1}, \ldots, s_{|Q|-1}$ be an enumeration of all the states of $G_{w, \Phi}$ different 


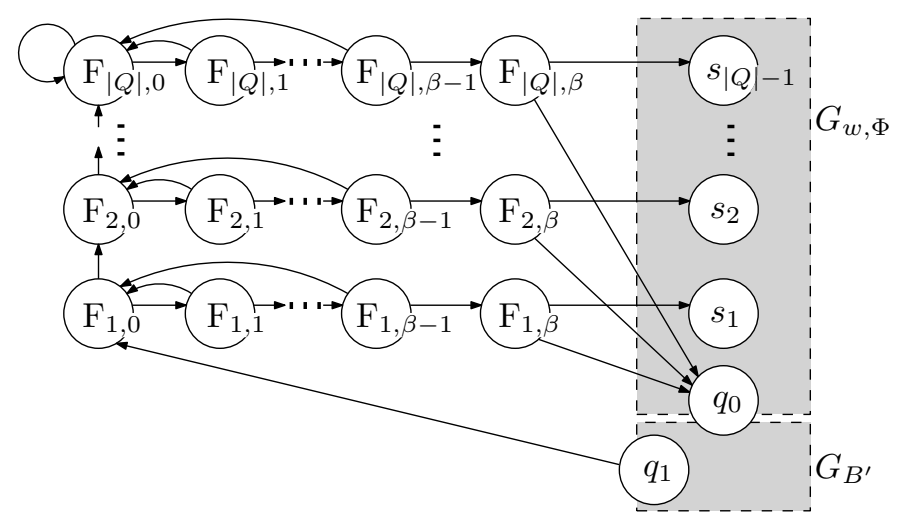

Fig. 6. The graph $\bar{G}_{w, \Phi}$

from $q_{0}^{\prime}$. Then we define $\bar{G}_{w, \Phi}$ as shown in Fig. 6] We merge the state $q_{0}^{\prime} \in Q$ with the state $q_{0} \in Q_{B}$, which is fixed by the definition of a sink device.

First, let there be a coloring $\bar{\delta}$ of $\bar{G}_{w, \Phi}$ such that $|\bar{\delta}(\bar{Q}, w)|=1$. It follows easily that $\bar{\delta}$, restricted to $Q$, encodes a coloring $\delta$ of $G_{w, \Phi}$ such that $|\delta(Q, w)|=1$. The choice of $G_{w, \Phi}$ guarantees that there is a satisfying assignment $\xi$ for $\Phi$.

On the other hand, let $\xi$ be a satisfying assignment of $\Phi$. By the choice of $G_{w, \Phi}$, there is a coloring $\delta$ of $G_{w, \Phi}$ such that $|\delta(Q, w)|=1$. We use the following coloring of $\bar{G}_{w, \Phi}$ : The edges outgoing from $s_{1}, \ldots, s_{|Q|-1}$ are colored according to $\delta$. The edges within $G_{B^{\prime}}$ are colored according to $B^{\prime}$. The edge $q_{1} \longrightarrow \mathrm{F}_{1,0}$ is colored by $b$. All the other edges incoming to the states $\mathrm{F}_{1,0}, \ldots, \mathrm{F}_{|Q|, 0}$, together with the edges of the form $\mathrm{F}_{i, \beta} \longrightarrow q_{0}$, are colored by $a$, while the remaining ones are colored by $b$.

For any $w \in\{a, b\}^{\star}$ we construct a strongly connected sink device $\mathbf{D}(w)=$ $\left(Q_{w},\{a, b\}, \delta_{w}\right)$. However, for some words $w \in T_{4}$ (e.g. for $\left.w=a b a b\right)$ the device $\mathbf{D}(w)$ is not incomplete and thus is not suitable for the reduction above. Take any $w \in\{a, b\}^{\star}$ and let $\mathfrak{C}_{w}^{\mathrm{P}}, \mathfrak{C}_{w}^{\mathrm{S}}, \mathfrak{C}_{w}^{\mathrm{F}}$ be the sets of all prefixes, suffixes and factors of $w$ respectively, including the empty word $\epsilon$. Let

$$
Q_{w}=\left\{[u] \mid u \in \mathfrak{C}_{w}^{\mathrm{F}}, v \notin \mathfrak{C}_{w}^{\mathrm{S}} \text { for each nonempty prefix } v \text { of } u\right\},
$$

while the partial transition function $\delta_{w}$ consists of the following transitions:

1. $[u] \stackrel{x}{\longrightarrow}[u x]$ whenever $[u],[u x] \in Q_{w}$,

2. $[u] \stackrel{x}{\longrightarrow}[\epsilon]$ whenever $u x \in \mathfrak{C}_{w}^{\mathfrak{S}}$,

3. $[u] \stackrel{x}{\longrightarrow}[\epsilon]$ whenever $[u x] \notin Q_{w}, u x \notin \mathfrak{C}_{w}^{\mathrm{S}}$, and $v x \in \mathfrak{C}_{w}^{\mathrm{P}}$ for a suffix $v$ of $u$.

Lemma 9. For any $w \in\{a, b\}^{\star}, \mathbf{D}(w)$ is a strongly connected sink device.

Lemma 10. Suppose that $w \in\{a, b\}^{\star}$ starts by $x$, where $\{x, y\}=\{a, b\}$. If there is $u \in\{a, b\}^{\star}$ satisfying all the following conditions, then $\mathbf{D}(w)$ is incomplete: 
1. $[u] \in Q_{w}$,

2. $u y \notin \mathfrak{C}_{w}^{\mathrm{F}}$,

3. for each nonempty suffix $v$ of uy, $v \notin \mathfrak{C}_{w}^{\mathrm{P}}$.

Theorem 11. If a word $w \in T_{4}$ satisfies some of the following conditions, then $\mathrm{SRCW}_{2,\{w\}}^{\mathcal{S C}}$ is NP-complete:

1. $w$ is of the form $w=x \bar{w} x$ for $\bar{w} \in\{a, b\}^{\star}, x \in\{a, b\}$,

2. $w$ is of the form $w=x \bar{w} y$ for $\bar{w} \in\{a, b\}^{\star}, x, y \in\{a, b\}, x \neq y$, and $x^{k} y^{l} x \in \mathfrak{C}_{w}^{\mathrm{F}}, x^{k+1} \notin \mathfrak{C}_{w}^{\mathrm{F}}, y^{l+1} \notin \mathfrak{C}_{w}^{\mathrm{F}}$ for some $k, l \geq 1$.

Proof. Due to Lemmas 8 and 9 , it is enough to show that $\mathbf{D}(w)$ is incomplete. Let $m \geq 1$ be the largest integer such that $y^{m}$ is a factor of $w$. It is straightforward to check that $u=y^{m}$ (in the first case) or $u=x^{k} y^{l}$ (in the second case) satisfies the three conditions from Lemma 10.

\section{Conclusion and Future Work}

We have completely characterized the binary words $w$ that make the computation of road coloring NP-complete if some of them is required to be the reset word for a coloring of a given graph. Except for $w=a^{k} b$ and $w=a^{k}$ with $k \geq 1$, each $w \in\{a, b\}^{\star}$ has this property. We have proved that if we require strong connectivity, the case $w=a b b$ becomes solvable in polynomial time. For any $w$ such that the first letter equals to the last one and both $a, b$ occur in $w$, we have proved that the NP-completeness holds even under this requirement. The main goals of the future research are:

- Complete the classification of binary words in the strongly connected case.

- Give the classifications of words over non-binary alphabets.

- Study SRCW restricted to non-singleton sets of words.

\section{References}

1. Adler, R., Goodwyn, L., Weiss, B.: Equivalence of topological Markov shifts. Israel Journal of Mathematics 27(1), 49-63 (1977)

2. Roman, A., Drewienkowski, M.: A complete solution to the complexity of synchronizing road coloring for non-binary alphabets (2014)

3. Roman, A.: P-NP threshold for synchronizing road coloring. In: Dediu, A.H., Martín-Vide, C. (eds.) Language and Automata Theory and Applications, Lecture Notes in Computer Science, vol. 7183, pp. 480-489. Springer Berlin Heidelberg (2012)

4. Schaefer, T.J.: The complexity of satisfiability problems. In: Proceedings of the Tenth Annual ACM Symposium on Theory of Computing. pp. 216-226. STOC '78, ACM, New York, NY, USA (1978)

5. Trahtman, A.N.: The road coloring and Černy conjecture. In: Stringology. pp. 1-12 (2008)

6. Vorel, V., Roman, A.: Parameterized complexity of synchronization and road coloring (2014) 